\title{
Osteo-necrosis of femoral head in North Indian population: Risk factors and clinico-radiological correlation
}

\author{
Tarun Goyal $^{\mathrm{a}, *}$, Anant Singh ${ }^{\mathrm{a}}$, Rahul Sharma ${ }^{\mathrm{b}}$, Arghya Kundu Choudhury ${ }^{\mathrm{b}}$, Shobha S. Arora ${ }^{\mathrm{a}}$

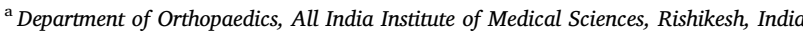 \\ ${ }^{\mathrm{b}}$ All India Institute of Medical Sciences, Rishikesh, India
}

\section{A R T I C L E I N F O}

\section{Keywords:}

Osteonecrosis

Avascular necrosis

Hip

Total hip replacement

\begin{abstract}
A B S T R A C T
Introduction: Osteonecrosis of femoral head (ONFH) is a common cause of destruction of hip joint in young patients and is one of the commonest indications of total hip replacement in India and other Asian countries. Data on risk factors for avascular necrosis and their relation to clinical and radiological outcomes is scarce. This study will help to understand the epidemiology of the condition and quantitatively define risk of disease with exposure to various known risk factors.

Materials and methods: 100 patients diagnosed to have ONFH and 100 matched controls were included in this prospective study. Details regarding alcohol consumption and steroid use were taken. Association between Harris hip score \& Visual analogue scale for pain and Ficat staging and area of femoral head involvement on MRI was studied. Association between amounts of alcohol and steroid consumed (average and cumulative doses) and clinical and radiological variables was also studied.

Results: Corticosteroid exposure was present in 44\%, alcohol in $30 \%$ patients and $22 \%$ cases were idiopathic. There was a statistically significant difference between average weekly alcohol consumption, drink-years, average daily dose of steroid and cumulative doses of steroids between the cases and the controls. Odds-ratio for steroid exposure in cases with ONFH was 134.5, 0.0006, and odds-ratio for alcohol exposure was 32.7, 0.0001. There was a significant correlation between the amount of alcohol $(r=-0.348, p=0.02)$ and steroid $(\mathrm{r}=-0.334, \mathrm{p}=0.001)$ consumption and HHS, and between amount of alcohol $(\mathrm{r}=0.312, \mathrm{p}=0.035)$ and steroid $(\mathrm{r}=0.546, \mathrm{p}=0.001)$ consumption and area of femoral head involvement. There was a strong positive correlation between clinical and radiological parameters (area of femoral head involved and HHS, $r=-0.70$, $\mathrm{p}=0.000)$.

Conclusion: Risk factors such as steroid intake and alcohol intake are important and accounts for majority of the cases of ONFH. The quantity of these exposures is directly related to the severity of disease on clinico-radiological scales.
\end{abstract}

\section{Introduction}

There is a huge disparity in indications for total hip replacement in Asian countries and western part of the globe. Osteonecrosis of femoral head (ONFH) is an uncommon indication of total hip replacement (THR) in USA and other western countries where osteoarthritis of hip is more common. ${ }^{1-3}$ Recent literature originating from Asian countries has shown that ONFH is far more common in these countries, accounting for about half of all THRs performed. ${ }^{4-7}$ Due to lack of multicentric joint registries in India, data on proportion of ONFH amongst patients undergoing THR is lacking. Nonetheless, it is generally agreed that $\mathrm{ONFH}$ is much more common in India than in the western world.
Major risk factors for development of ONFH include steroid consumption, alcohol use or trauma to the hip joint. ${ }^{1,5,6}$ Data on risk factors for avascular necrosis in Indian patients is not known. Knowledge of risk factors of ONFH may help to identify patients at risk of developing avascular necrosis. This is important because natural history of untreated osteonecrosis of femoral head is dismal. ${ }^{8}$ Majority of these will eventually progress to significant collapse and secondary degenerative arthritis. By the time most patients come to clinical attention, significant collapse of the femoral head has already occurred.

The aim of this study is to study relative prevalence of various known risk factors of ONFH in population from North India. Correlations between amount of exposure, clinical and radiological

\footnotetext{
* Corresponding author. Department of Orthopaedics, AIIMS, Virbhadra Marg, Rishikesh, 248201, India.

E-mail addresses: goyal.tarun@gmail.com (T. Goyal), anantsingh1305@gmail.com (A. Singh), rscoolsharma94@gmail.com (R. Sharma), arghyakunduchoudhury@gmail.com (A.K. Choudhury), drshobhaarora@gmail.com (S.S. Arora).
} 
severity of the disease will also be studied.

\section{Material and methods}

A convenience sample of all adult patients (more than 18 years), of either sex, presenting to orthopaedic clinic between July 2016December 2017, and diagnosed to have ONFH was taken for study. Since this is the first study correlating alcohol and steroid intake and clinic-radiological parameters, and no reference of coefficient coefficients is available in literature, sample size calculation could not be done. Informed consent was obtained from each participant prior to the study. Ethics approval for the study had been taken from the ethics committee of the institute. All these patients had undergone anteroposterior and lateral radiographs and magnetic resonance imaging (MRI) of the hip joint. Definite diagnosis of avascular necrosis was made on the basis of characteristic MRI findings. Patients having symptoms for more than 5 years were excluded from the study to reduce risk of recall bias. The retained participants were interviewed during their clinical visit about various risk factors for ONFH in last 5 years. Details regarding alcohol consumption and steroid use were taken. Type, amount and total duration of alcohol use was taken from the subjects. Average weekly amount of alcohol consumed was calculated in terms of units of ethanol consumed per week. Multiplying average weekly consumption of liquor in millilitres by the percentage of ethanol contained in the liquor gives the daily ethanol consumption. One alcohol unit is measured as $10 \mathrm{ml}$ of pure ethanol. This equals one $25 \mathrm{ml}$ single measure of whisky, vodka, rum, country-liquor (average alcohol content $40 \%$ ) or a third of a pint of beer (average alcohol content $5-6 \%)$. Use of locally made country-liquor is also common in this region and the percentage of its alcohol content is not standardised and may vary from $35 \%$ to $45 \%$ according to local standards. The alcohol content was taken as $40 \%$ for this study. Drink-years were calculated as number of units consumed per week multiplied by the number of years of consumption. Since unlike corticosteroid exposure, a large proportion of normal population would be exposed to alcohol because of social drinking a cut-off limit for alcohol exposure was defined. Patient was considered to be exposed to alcohol if the weekly consumption was more than 21 units, which equalled to about 3 units consumed on most days. Units of alcohol consumed per day and drinkyears of alcohol consumption were correlated with pre-operative Harris Hip Score (HHS), Visual analogue scale (VAS) for pain from 1 to 10 with 10 being the worst score, radiological stage of disease and area of involvement of the femoral head. HHS is a frequently used outcome measure in hip disorders. It measures pain, function, deformity and range of motion and scores range between 0 and 100, with higher scores meaning a better hip function.

Similarly, details on dose, drug, route and duration of administration were taken for steroid use. The amount of different steroids consumed were converted to equivalent doses of prednisolone consumed. ${ }^{9}$ Daily doses of steroid used and cumulative doses of steroid consumed over time were correlated with HHS, VAS score for pain, radiological stage of disease and area of involvement of the femoral head. History of significant trauma to the hip joint leading to fractures or dislocations were noted. Type of injury, treatment given and duration since injury were noted. Patients not having exposure to any of these studied known risk factors were classified as idiopathic group. Before labelling a patient as having idiopathic avascular necrosis other risk factors such as sickle cell disease, coagulopathies, Caissons disease, Gaucher's disease, pregnancy or oral contraceptive use were excluded on the basis of history, physical examination and previous medical records.

The clinical examination findings, Harris Hip score (HHS) and visual analogue scale (VAS) score for pain were noted. Radiological grade of ONFH on plain radiographs was noted according to the staging given by Ficat. ${ }^{10}$ The percentage of femoral head involvement by the necrotic lesions was measured on magnetic resonance images (MRI) using technique described by Kim et al. ${ }^{11}$ We measured the largest medio- lateral diameter of the femoral head $(\mathrm{R})$ and the longest medio-lateral length of the necrotic lesion (A) on coronal magnetic resonance images and the largest antero-posterior diameter of the femoral head (S) and the longest antero-posterior length of the necrotic lesion (B) on sagittal magnetic resonance images. The two-dimensional extent of a necrotic lesion was determined with the equation: $\%$ area $=\left[\left(\mathrm{A}^{*} \mathrm{~B}\right) /\right.$ $(\mathrm{R} * \mathrm{~S})] * 100$.

One control was identified for each case included, which was matched for age, sex and BMI. These controls were asymptomatic voluntary participants, preferably the people accompanying the patients to hospital, who had no complaints in the hip joint, no prior history of hip involvement and normal examination findings on clinical examination of the hip joint. Details of steroid and alcohol consumption were compared between the cases and the controls.

Three different observers were involved in the study, one for questioning the patients about risk factors for avascular necrosis, one for noting VAS score and HHS and lastly one for noting the radiological findings. They were blinded to all other parameters except what they were studying. Correlation between HHS, VAS and area of femoral head involvement on MRI was made using Spearman correlation coefficient. Association between HHS, VAS for pain and Ficat staging was calculated using ANOVA. Student T test was used to study association between the continuous variables. Test for normality was done using Normal Q-Q Plots. Fisher's exact test was used for studying association between for FICAT stage and types of ONFH as some values in each category were less than five. SPSS software version 17.0 (SPSS Inc., Chicago, IL, USA) was used for all statistical calculations.

\section{Results}

A total of 100 patients diagnosed with ONFH presented to our clinic and were included in the study. Demographic details of the patients, details of risk factors such as corticosteroid use, alcohol consumption and clinical and radiological outcomes are shown in Table 1. Corticosteroid exposure was present in $44(44 \%)$ patients, alcohol exposure was present in $30(30 \%)$ of the patients and $22(22 \%)$ cases were classified as idiopathic. Four patients had post-traumatic ONFH (nonunion neck of femur in 2 patients and acetabulum fracture in 2 patients). Bilateral involvement of the hip joint was seen in 63 patients. Bilateral involvement was seen in $33(75 \%)$ patients in the corticosteroid group, 20 (67\%) patients in alcohol group and 10 (45\%) patients in idiopathic group. There was no statistically significant difference in mean age, sex, height, weight or duration of symptoms between different groups. There was no significant difference between the groups in VAS score for pain, HHS or percentage area of femoral head involvement. Patients with steroid induced and alcohol induced ONFH had a significantly higher proportion of patients with collapse of femoral head using Ficat staging system.

The common indications for corticosteroid use were rheumatoid arthritis $(n=17)$, ankylosing spondylitis $(n=9)$ bronchial asthma $(n=7)$ and psoriasis $(n=2)$. Nine patients had taken anabolic steroids without any formal medical prescriptions. Average and cumulative exposure to steroids and alcohol is summarised in Table 2. There was a statistically significant difference between average weekly alcohol consumption, drink-years, average daily dose of steroid and cumulative doses of steroids between the cases and the controls. A significant correlation was seen between the amount of alcohol and steroid consumption and VAS score for pain, HHS and percentage area of femoral head involvement (Table 3). There was a significant association between the amount of alcohol and steroid consumption and Ficat stage (Table 3). There was a strong correlation between percentage area of femoral head involved VAS score for pain and HHS. Patients with larger area of femoral head involvement had greater VAS scores and lower HHS. Association between Ficat stage and VAS score for pain and HHS was also statistically significant (Table 4). Odds ratios for exposure to alcohol and steroids are shown in Table 5. 
Table 1

Demographic data, clinical and radiological findings in different sub-groups of patients with ONFH (n-100).

\begin{tabular}{|c|c|c|c|c|c|}
\hline & Corticosteroid exposure & Alcohol exposure & No exposure to studied risk factors & Post-traumatic & $P$ value \\
\hline Number of patients & 44 & 30 & 22 & 4 & - \\
\hline Number of hips & 77 & 50 & 32 & 4 & - \\
\hline Mean age (SD) & $32.4(12.6)$ & $37.3(15.6)$ & $42.6(18.2)$ & $30.6(10.1)$ & 0.06 \\
\hline Sex (Male: Female) & $34: 10$ & 30 males, no females & $13: 9$ & $3: 1$ & 0.8 \\
\hline Mean height in $\mathrm{cm}$ (SD) & 161 & 168 & 164 & 167 & 0.45 \\
\hline Mean weight in $\mathrm{Kg}(\mathrm{SD})$ & 58 & 70 & 62 & 68 & 0.09 \\
\hline Mean duration of symptoms (months) & 4.5 & 6.3 & 5.7 & 7.6 & 0.08 \\
\hline $\begin{array}{l}\text { VAS score for pain } \\
\text { Median (inter-quartile range) }\end{array}$ & $8.4(2.1)$ & $8.1(2.2)$ & $7.1(1.8)$ & $7.3(2.0)$ & 0.15 \\
\hline HHS & 54.6 & 56.3 & 60.5 & 62.4 & 0.42 \\
\hline Ficat stage & & & & & 0.04 \\
\hline I & 5 & 4 & 3 & 0 & \\
\hline II & 12 & 9 & 9 & 0 & \\
\hline III & 36 & 25 & 15 & 2 & \\
\hline IV & 24 & 12 & 5 & 2 & \\
\hline Mean \% area of femoral head involved & $62 \%(27.5)$ & $57 \%(36.2)$ & $45 \%(18.4)$ & $58 \%(22.2)$ & 0.17 \\
\hline
\end{tabular}

Table 2

Comparison of exposure to steroids and alcohol between the cases and controls (n-100).

\begin{tabular}{|c|c|c|c|}
\hline & Cases & Control & $P$ value \\
\hline Weekly units of alcohol consumed (SD) & $16.6(4.3)$ & $6.5(3.6)$ & 0.000 \\
\hline Drink-years (SD) & $324(24.8)$ & $88(18.3)$ & 0.000 \\
\hline $\begin{array}{l}\text { Daily dose of corticosteroids (prednisolone } \\
\text { equivalent in mg) used (SD) }\end{array}$ & $32(6.6)$ & $.8(0.4)$ & 0.000 \\
\hline $\begin{array}{l}\text { Cumulative dose of corticosteroids } \\
\text { (prednisolone equivalent in mg) used } \\
\text { (SD) }\end{array}$ & $\begin{array}{l}17520 \\
(506.4)\end{array}$ & $48(26.8)$ & 0.000 \\
\hline
\end{tabular}

\section{Discussion}

ONFH leads to significant disability in the most productive years of life and is one of the common causes of hip arthroplasty in younger individuals. Literature is lacking on prevalence of various known risk factors for ONFH from India. This is the first study evaluating clinicradiological correlation between symptoms, functional scores and radiological scoring in ONFH. Exposure to steroid and alcohol were strongly associated with ONFH in this study. Steroid exposure was the most common risk factor for ONFH in our series, present in $44 \%$ of the patients. This was followed by exposure to alcohol, which was seen in $30 \%$ of the patients. Together these amount to about three-fourth of all patients with ONFH.

We observed a positive correlation between the amounts of steroids and alcohol consumed and clinical and radiological scores. Larger amounts of alcohol and steroid consumptions were associated with worse HHS, higher pain scores and larger areal involvement of humeral heads. Correlation between the clinical outcomes and radiological scores was also strong. Association between ONFH and steroid exposure is well established in literature. ${ }^{12}$ Though exact mechanism is not known various theories have suggested role of increased adipocyte volume, increased intra-osseous pressure and fat embolism as final
Table 4

Correlation between functional outcomes (measured by HHS and VAS for pain) and radiological outcomes (measured by Ficat stage and percentage area of femoral head involved) (n-100).

\begin{tabular}{lll}
\hline & HHS & VAS for pain \\
\hline Ficat stage & $\mathrm{p}=0.002$ & 0.015 \\
Mean \% area of femoral head involved & $\mathrm{r}=-0.70$ & $\begin{array}{l}\mathrm{r}=0.63 \\
\mathrm{p}=0.000\end{array}$ \\
& $\mathrm{p}=0.001$ \\
\hline
\end{tabular}

Table 5

Odds ratios for exposure to alcohol and steroids $(n=100)$.

\begin{tabular}{lll}
\hline & Number & Odds ratio, p-value \\
\hline Cases exposed to alcohol & 30 & $32.7,0.0001$ \\
Controls exposed to alcohol & 2 & $134.5,0.0006$ \\
Cases exposed to steroids & 44 & \\
Controls exposed to steroids & 0 & \\
\hline
\end{tabular}

mechanisms. $^{13}$

Tsai et al. ${ }^{14}$ from Taiwan found that alcohol consumption was the most common etiology contributing to $45.2 \%$ cases of ONFH, followed by idiopathic AVN in $33.1 \%$ and steroid induced AVN in $21.7 \%$. In total $59.1 \%$ cases were bilateral. Kang et al. ${ }^{6}$ from Korea found that $32.4 \%$ patients with ONFH had history of alcohol abuse, and $14.6 \%$ were exposed to steroid. Bilateral involvement was noticed in $37 \%$. Fukushima et al. ${ }^{5}$ from Japan found a higher incidence of steroid intake (51\%) and alcohol abuse (31\%) in their population of patients with ONFH. This was closer to our findings of $44 \%$ steroid induced and $30 \%$ alcohol induced ONFH. In the study by Castro et al. ${ }^{15}$ mean age of alcohol induced AVN was significantly higher compared to steroid induced or idiopathic ONFH. There was also higher incidence of collapse in patients with alcohol induced ONFH (90\%) compared to steroid induced (62\%) or idiopathic ONFH (55\%). We found that the age of idiopathic

Table 3

Correlation/association between amounts of alcohol and steroid consumption and clinical and radiological outcomes (n-100).

\begin{tabular}{lllll}
\hline & HHS & VAS for pain & Mean \% area of femoral head involved & Ficat stage \\
\hline Average weekly consumption of alcohol & $\mathrm{r}=-0.222$ & $\mathrm{r}=0.082$ & $\mathrm{r}=0.246$ & $\mathrm{p}=0.002$ \\
Drink-years of alcohol & $\mathrm{p}=0.08$ & $\mathrm{p}=0.37$ & $\mathrm{p}=0.025$ & $\mathrm{r}$ \\
& $\mathrm{r}=-0.348$ & $\mathrm{r}=0.234$ & $\mathrm{r}=0.312$ & $\mathrm{p}=0.012$ \\
Daily dose of corticosteroids used & $\mathrm{p}=0.02$ & $\mathrm{p}=0.048$ & $\mathrm{p}=0.035$ & $\mathrm{p}=0.030$ \\
& $\mathrm{r}=-0.182$ & $\mathrm{r}=0.102$ & $\mathrm{r}=0.322$ & $\mathrm{p}$ \\
Cumulative dose of corticosteroids consumed & $\mathrm{p}=0.077$ & $\mathrm{p}=0.035$ & $\mathrm{p}=0.06$ & $\mathrm{p}=0.015$ \\
& $\mathrm{r}=-0.334$ & $\mathrm{r}=0.252$ & $\mathrm{p}=0.001$ & \\
& $\mathrm{p}=0.001$ & $\mathrm{p}=0.015$ & & \\
& & &
\end{tabular}


ONFH was higher than steroid or alcohol associated ONFH. We found a higher incidence of collapse in both steroid and alcohol induced ONFH (Table 1). Total proportion of bilateral cases was also higher in our series compared to other studies. ${ }^{5,6}$ Also the proportion of cases with bilateral involvement was higher in steroid and alcohol induced ONFH. We also found a positive correlation between amounts of these exposures and clinic-radiological involvement.

This study has several limitations. Cut-off values for alcohol consumption and steroid exposure in causing ONFH have not been studied yet. Since social consumption of alcohol is otherwise common in most cultures world-wide, a definition of heavy alcohol use in occurrence of ONFH has to be studied. We took a cut-off value of weekly consumption of more than 21 units of alcohol. Since this is the first study correlating alcohol and steroid intake and clinic-radiological parameters, and no reference of coefficient coefficients is available in literature sample size calculation could not be done. Many lacunas need to be addressed on pathogenesis and outcome of ONFH. ${ }^{16}$ There is a need of long term studies on patients with ONFH focussing on natural history of the disease and survival analysis with THR as end point. Differences in incidence, risk factors and outcomes of ONFH in different populations also points towards possibility of genetic differences or specific environmental exposures.

\section{Conclusion}

Risk factors such as steroid intake and alcohol intake are important and accounts for majority of the cases of ONFH. Steroid intake has emerged as the most common factor associated with ONFH in this study. The quantity of these exposures is directly related to the severity of disease on clinic-radiological scales. People having these exposures should be counselled regarding risk of ONFH, and quantity of these exposures reduced as far as possible. Surveillance of ONFH in these patients may help diagnosis of these cases before advance collapse of femoral head has occurred, providing an opportunity of treatment to these patients.

\section{Author Contribution}

Dr Tarun Goyal: Planning of study, patient enrolment, writing of manuscript. Dr. Anant Singh: Evaluation of functional outcomes, data management. Dr Rahul Sharma: Evaluation of functional outcomes, data management. Dr Arghya Kundu Choudhury: Evaluation of functional outcomes, data management, manuscript preparation. Prof Shobha S. Arora: Planning of study, patient enrolment, writing of manuscript.

\section{Conflicts of interest}

The authors declare that they have no conflict of interest.

\section{Informed consent}

Informed consent was obtained from all individual participants included in the study.

\section{Ethics approval}

Ethics approval for the study had been taken from the ethics committee of the institute before starting the study.

\section{Appendix A. Supplementary data}

Supplementary data to this article can be found online at https:// doi.org/10.1016/j.cegh.2019.01.012.

\section{References}

1. Vail TP, Covington DB. The incidence of osteonecrosis. In: Urbaniak JR, Jones JP, Jreds. Osteonecrosis: Etiology, Diagnosis, Treatment. Rosemont, IL: American Academy of Orthopedic Surgeons; 1997:43-49.

2. Havelin L. The Norwegian joint registry. Bull Hosp Jt Dis. 1999;58:139-147.

3. Malchau H, Herberts P, Eisler T, Garellick G, Soderman P. The Swedish total hip replacement register. J Bone Joint Surg Am. 2002;84-A:2-20.

4. Lai YS, Wei HW, Cheng CK. Incidence of hip replacement among national health insurance enrollees in Taiwan. J Orthop Surg Res. 2008;3:42.

5. Fukushima W, Fujioka M, Kubo T, Tamakoshi A, Nagai M, Hirota Y. Nationwide epidemiologic survey of idiopathic osteonecrosis of the femoral head. Clin Orthop Relat Res. 2010;468:2715-2724

6. Kang JS, Park S, Song JH, Jung YY, Cho MR, Rhyu KH. Prevalence of osteonecrosis of the femoral head: a nationwide epidemiologic analysis in Korea. $J$ Arthroplasty. 2009;24:1178-1183.

7. Tang WM, Chiu KY. Avascular necrosis of femoral head: a short review. APLAR J Rheumatol. 2006;9:98-101.

8. Tripathy SK, Goyal T, Sen RK. Management of femoral head osteonecrosis: current concepts. Indian J Orthop. 2015;49(1):28-45.

9. Mager DE, Lin SX, Blum RA, Lates CD, Jusko WJ. Dose equivalency evaluation of major corticosteroids: pharmacokinetics and cell trafficking and cortisol dynamics. $J$ Clin Pharmacol. 2003;43(11):1216-1227.

10. Ficat RP. Idiopathic bone necrosis of the femoral head. Early diagnosis and treatment. J Bone Joint Surg. 1985;67:3-9.

11. Kim YM, Ahn JH, Kang HS, Kim HJ. Estimation of the extent of osteonecrosis of the femoral head using MRI. J Bone Joint Surg Br. 1998;80:954-958.

12. M1 Sakaguchi, Tanaka T, Fukushima W, Kubo T, Hirota Y. Idiopathic ONF Multicenter Case-Control Study Group. Impact of oral corticosteroid use for idiopathic osteonecrosis of the femoral head: a nationwide multicenter case-control study in Japan. J Orthop Sci. 2010;15(2):185-191.

13. Wang GJ1, Cui Q, Balian G. The Nicolas Andry award. The pathogenesis and prevention of steroid-induced osteonecrosis. Clin Orthop Relat Res. 2000;370:295-310.

14. Tsai SW, Wu PK, Chen CF, et al. Etiologies and outcome of osteonecrosis of the femoral head: etiology and outcome study in a Taiwan population. J Chin Med Assoc. 2015;79(1):39-45.

15. Castro Jr FP, Harris MB. Differences in age, laterality, and Steinberg stage at initial presentation in patients with steroid-induced, alcohol-induced, and idiopathic femoral head osteonecrosis. J Arthroplasty. 1999;14(6):672-676.

16. Mont MA1, Cherian JJ, Sierra RJ, Jones LC, Lieberman JR. Nontraumatic osteonecrosis of the femoral head: where do we stand today? A ten-year update. $J$ Bone Joint Surg Am. 2015;97(19):1604-1627.

\section{Funding}

There is no funding source. 